\title{
Neutrino Physics and Astrophysics with the MACRO Experiment at the Gran Sasso Lab
}

\author{
Giorgio Giacomelli* \\ Dipartimento di Fisica dell'Università di Bologna \\ and INFN, Sezione di Bologna, \\ it I-40127 Bologna, Italy
}

Received on 1 October, 2002

\begin{abstract}
The results of the MACRO experiment on atmospheric neutrino oscillations are presented and discussed. The data concern different event topologies with average neutrino energies of $\sim 4$ and $\sim 50 \mathrm{GeV}$. The Multiple Coulomb Scattering of muons in the MACRO absorbers was used to estimate the neutrino energy of each event of the higher energy sample. The angular distributions, the absolute fluxes and the L/E distribution strongly favour $\nu_{\mu} \rightarrow \nu_{\tau}$ oscillations with maximal mixing and $\Delta m^{2}=0.0025 \mathrm{eV}^{2}$. Results are presented on the searches for astrophysical sources of high energy muon neutrinos, for bursts of electron antineutrinos from stellar gravitational collapses and on indirect searches for WIMPs from the Earth and from the Sun.
\end{abstract}

\section{Introduction}

MACRO was a large multipurpose area underground experiment designed to search for rare events in the penetrating cosmic radiation. These included the study of atmospheric neutrinos and their oscillations, high energy $\left(E_{\nu} \gtrsim 1 \mathrm{GeV}\right)$ muon neutrino astronomy, indirect searches for WIMPs and search for low energy $\left(E_{\nu} \gtrsim 7 \mathrm{MeV}\right)$ stellar collapse neutrinos. The detector was placed in the Gran Sasso laboratory, located on the highway Rome-Teramo , $120 \mathrm{~km}$ east of Rome. The lab consists of three underground halls, each about $100 \mathrm{~m}$ long. It is at an altitude of $963 \mathrm{~m}$ above sea level, is well shielded from cosmic rays by a mean rock thickness of $\simeq 3700$ m.w.e.; the minimum is 3150 m.w.e: this defines the minimum muon energy at the surface at $\sim 1.3 \mathrm{TeV}$ in order to reach MACRO. The average residual muon energy and the muon flux are $\sim 320 \mathrm{GeV}$ and $\sim 1 \mathrm{~m}^{-2} \mathrm{~h}^{-1}$, respectively.

MACRO was composed of three sub-detectors: liquid scintillation counters, limited streamer tubes and nuclear track detectors. Each one of them could be used in "standalone" and in "combined" mode. A cross section of the detector is shown in Fig. 1. Notice the division in the lower and in the upper part (this was often referred to as the Attico); the inner part of the Attico was empty and lodged the electronics [1]. The mass of the lower MACRO was $\simeq 4200 \mathrm{t}$, mainly in the form of boxes filled with crushed Gran Sasso rock. The detector had a modular structure: it was divided into six sections referred to as supermodules. Each active part of one supermodule had a size of $12.6 \times 12 \times 9.3 \mathrm{~m}^{3}$ and had a separate mechanical struc- ture and electronics readout. The full detector had global dimensions of $76.5 \times 12 \times 9.3 \mathrm{~m}^{3}$ and provided a total acceptance to an isotropic flux of particles of $\sim 10000 \mathrm{~m}^{2} \mathrm{sr}$. The total mass was $\simeq 5300 \mathrm{t}$.

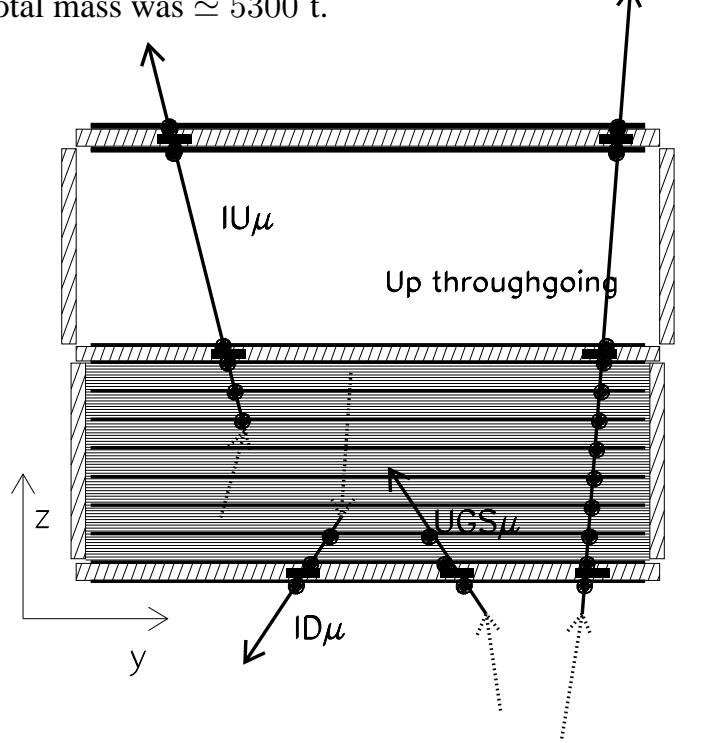

Figure 1. Cross section of the MACRO detector and sketch of different event topologies induced by $\nu_{\mu} \rightarrow \mu$ charged current interactions. The black points and the black rectangles represent streamer tubes and scintillator hits, respectively. Tracking was performed by the streamer tubes; the time-of-flight (T.O.F.) of the muons was measured by the scintillators for Up Semicontained (Internal upgoing - IU $\mu$ ) and Upthroughgoing events (and also for downgoing muons).

Data were taken from March 1989 till april 1994 with the detector under construction and from the middle of 1994

\footnotetext{
*MACRO Collaboration. See Ref. 1 for a list of MACRO Collaborators and Institutions.
} 
till the end of 2000 with the full detector.

\section{Atmospheric neutrino oscillations}

If neutrinos have non-zero masses, one has to consider the weak flavour eigenstates $\nu_{\mathrm{e}}, \nu_{\mu}, \nu_{\tau}$ and the mass eigenstates $\nu_{1}, \nu_{2}, \nu_{3}$. The weak flavour eigenstates $\nu_{l}$ are linear combinations of the mass eigenstates $\nu_{\mathrm{m}}$ through the elements of the mixing matrix $U_{l m}$ :

$$
\nu_{l}=\sum_{m=1}^{3} U_{l m} \nu_{m}
$$

In the simple case of only two flavour eigenstate neutrinos $\left(\nu_{\mu}, \nu_{\tau}\right)$ which oscillate with two mass eigenstates $\left(\nu_{2}, \nu_{3}\right)$ one has

$$
\left\{\begin{array}{l}
\nu_{\mu}=\nu_{2} \cos \theta_{23}+\nu_{3} \sin \theta_{23} \\
\nu_{\tau}=-\nu_{2} \sin \theta_{23}+\nu_{3} \cos \theta_{23}
\end{array}\right.
$$

where $\theta_{23}$ is the mixing angle ( $\theta$ will be used in the following). In this case one may easily compute the following expression for the survival probability of a $\nu_{\mu}$ beam

$$
P\left(\nu_{\mu} \rightarrow \nu_{\mu}\right)=1-\sin ^{2} 2 \theta_{23} \sin ^{2}\left(\frac{E_{2}-E_{1}}{2} t\right)=1-\sin ^{2} 2 \theta_{23} \sin ^{2}\left(\frac{1.27 \Delta m^{2} \cdot L}{E_{\nu}}\right)
$$

where $\Delta m^{2}=\mathrm{m}_{3}^{2}-\mathrm{m}_{2}^{2}$ and $\mathrm{L}$ is the distance travelled by the muon neutrino from production to detection.

High energy primary cosmic rays interact in the upper atmosphere producing pions and Kaons, which by decay yield muons and muon neutrinos, $\pi \rightarrow \mu \nu_{\mu}$; further decays of the muons lead to electron and muon neutrinos, $\mu \rightarrow \nu_{\mu} \nu_{l} l$. The neutrinos are produced in a spherical shell at about $10-20 \mathrm{~km}$ from the earth surface. Upgoing $\nu_{\mu}$ 's may interact in the rock below MACRO or inside its lower part leading to upgoing muons, $\nu_{\mu} N \rightarrow \mu^{+-}+\ldots$.

Upward going muons are identified by the streamer tube system (for tracking) and the scintillator system (for timeof-flight measurement). A rejection factor of at least $10^{7}$ is needed in order to separate upgoing muons from the background due to the downgoing muons. Fig. 1 shows sketches of the different neutrino event topologies analyzed: Upthroughgoing muons, Upsemicontained (also called Internal Upgoing muons, IU), Upgoing Stopping muons (UGS), Internal Downgoing muons (ID). The numbers of events measured and expected for the three topologies are given in Table . All data samples deviate from Monte Carlo expectations; the deviations point to neutrino oscillations.

\section{II.1 Upthroughgoing muons}

The upthroughgoing muons come from $\nu_{\mu}$ interactions in the rock below the detector; the $\nu_{\mu}$ 's have an average energy $\bar{E}_{\nu} \sim 50 \mathrm{GeV}[2,3]$. The data refer to a lifetime of 6.16 years (full detector equivalent). The data deviate in absolute value and in shape from Monte Carlo (MC) predictions, see Fig. 2. We studied a large number of possible effects that could affect our measurements: no significant systematic problems exist in the detector or in the data analyses.

The measured data have been compared with MC simulations. For the upthroughgoing muon simulation, the neutrino flux computed by the Bartol group was used [4]. The cross sections for the neutrino interactions were calculated using the deep inelastic parton distributions of ref. [5]. The muon propagation to the detector was done using the energy loss calculation in standard rock. The total systematic uncertainty on the expected muon flux, obtained adding in quadrature the errors from neutrino flux, cross section and muon propagation, is estimated to be $17 \%$. This uncertainty is mainly a scale error; the error on the shape of the angular distribution is $\sim 5 \%$.

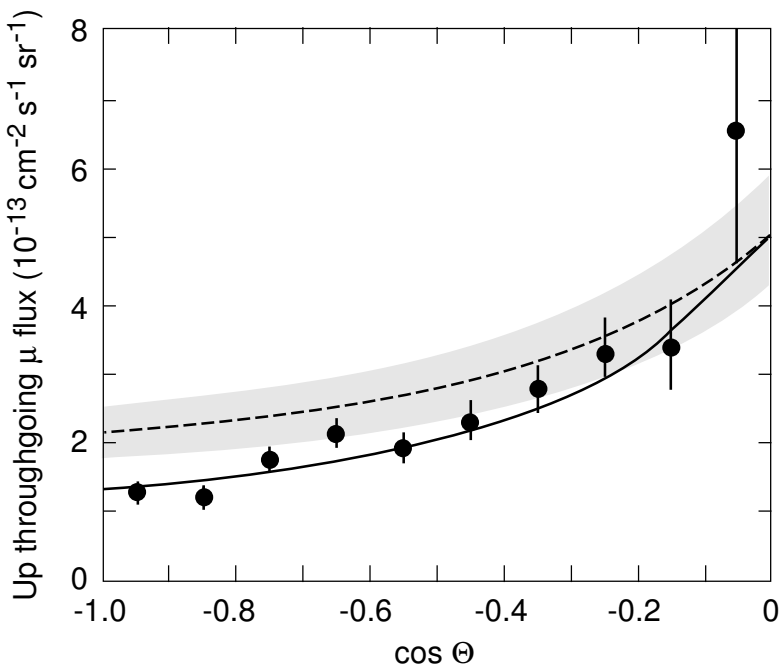

Figure 2. Zenith angle distribution of upthroughgoing muons (black points). The dashed line is the expectation for no oscillations (with a $17 \%$ scale uncertainty band). The solid line is the fit for an oscillated muon flux which yields maximum mixing and $\Delta m^{2}=2.5 \cdot 10^{-3} \mathrm{eV}^{2}$ [2] [3].

Figure 2 shows the zenith angle $(\Theta)$ distribution of the measured flux of upthroughgoing muons. The Monte Carlo expectation for no oscillations is shown as a dashed line. Assuming $\nu_{\mu} \rightarrow \nu_{\tau}$ oscillations, the best fit parameters are $\Delta m^{2}=2.5 \cdot 10^{-3} \mathrm{eV}^{2}$ and $\sin ^{2} 2 \theta=1$ with a probability of $66 \%$; the result of the fit is the solid line in Fig. 2. The probability for no-oscillations is $0.4 \%$. 
Figure 3 shows the allowed region for $\nu_{\mu} \rightarrow \nu_{\tau}$ oscillations in the $\sin ^{2} 2 \theta-\Delta m^{2}$ plane, computed according to ref. [6] for the upthroughgoing muon events; our region is compared with those obtained by the SuperKamiokande (SK) [7] and Soudan $2[8]$ experiments.

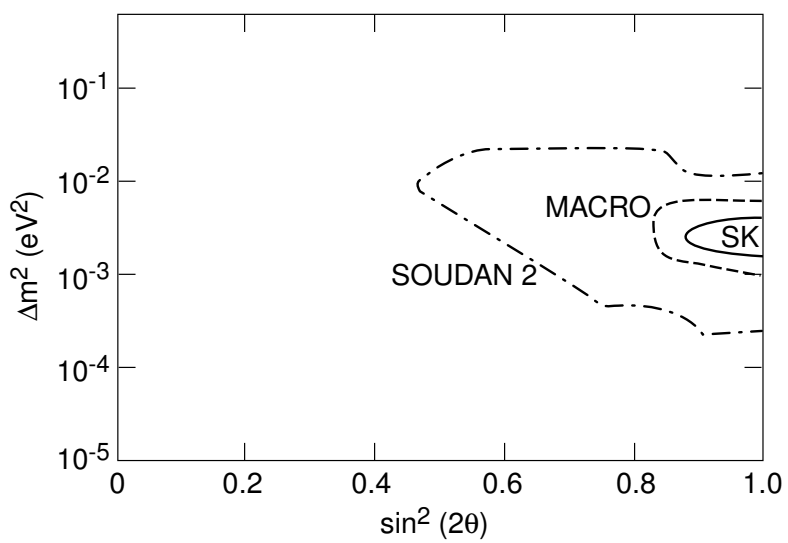

Figure $3.90 \%$ c.l. allowed regions for $\nu_{\mu} \rightarrow \nu_{\tau}$ oscillations from upthroughgoing muons and comparison with the Soudan 2 and SK allowed regions (the Soudan 2 region is now smaller).

II.2 Matter effects. $\nu_{\mu} \rightarrow \nu_{\tau}$ versus $\nu_{\mu} \rightarrow \nu_{\text {sterile }}$

Matter effects due to the difference between the weak interaction effective potential for muon neutrinos with respect to sterile neutrinos would produce a different total number and a different zenith distribution of upthroughgoing muons [9]. In Fig. 4 the measured ratio between the events with $-1<\cos \Theta<-0.7$ and the events with $-0.4<\cos \Theta<0$ is shown as a black point. In this ratio most of the theoretical uncertainties on neutrino flux and cross section cancel. The remaining theoretical error combined with the experimental error is estimated to be $7 \%$. We measured 305 events with $-1<\cos \Theta<-0.7$ and 206 with $-0.4<\cos \Theta<0$; the ratio is $\mathrm{R}=1.48 \pm 0.13_{\text {stat }} \pm 0.10_{\text {sys }}$. For $\Delta m^{2}=2.5 \cdot 10^{-3} \mathrm{eV}^{2}$ and maximal mixing, the expected value of the ratio for $\nu_{\mu} \rightarrow \nu_{\tau}$ is $R_{\tau}=1.72$ while for $\nu_{\mu} \rightarrow \nu_{s}$ is $R_{\text {sterile }}=2.16$. The maximum probabilities $P_{\text {best }}$ to find a value of $R_{\tau}$ and of $R_{\text {sterile }}$ smaller than the expected ones are $9.4 \%$ and $0.06 \%$ respectively. The ratio

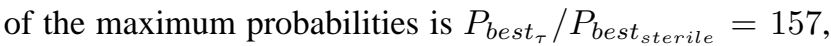
so that $\nu_{\mu} \rightarrow \nu_{s}$ oscillations are disfavoured at $99 \%$ c.l. compared to the $\nu_{\mu} \rightarrow \nu_{\tau}$ channel $[3,9]$.

\section{II.3 $\nu_{\mu}$ energy estimates by MCS of upthrough- going muons}

The oscillation probability is a function of the ratio $L / E_{\nu}$, Eq. 3. Even if a precise measurement of $E_{\nu}$ is not possible, MC simulations have shown a correlation between $E_{\nu}$ and the residual muon energy $E_{\mu} . E_{\nu}$ was thus estimated for each event by measuring the muon energy $E_{\mu}$, via Multiple Coulomb Scattering (MCS) of the iduced muon in

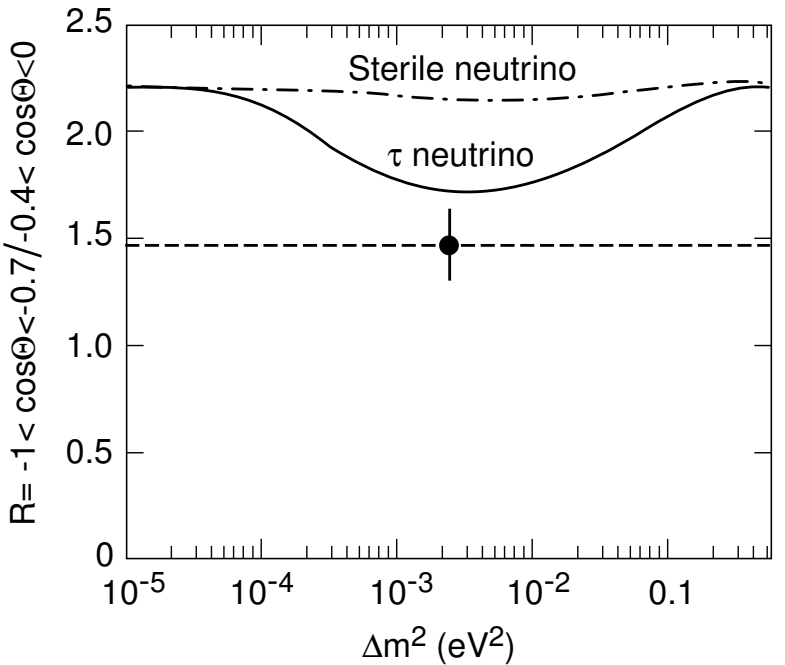

Figure 4. Ratio of events with $-1<\cos \theta<-0.7$ to events with $-0.4<\cos \theta<0$ vs $\Delta m_{23}^{2}$ for maximal mixing. The black point is the measured value, the solid line is the prediction for $\nu_{\mu} \rightarrow \nu_{\tau}$ oscillations, the dash-dotted line is the prediction for $\nu_{\mu} \rightarrow \nu_{\text {sterile }}$ oscillations.

the absorbers. The r.m.s. of the lateral displacement for a muon crossing the apparatus on the vertical is $\sigma_{M C S} \simeq$ $10 \mathrm{~cm} / \mathrm{E}_{\mu}(\mathrm{GeV})$. The muon energy $E_{\mu}$ can be measured up to a saturation point, occurring when $\sigma_{M C S}$ is comparable with the detector space resolution. A first analysis was made studying the deflection of upthroughgoing muons with the streamer tubes in "digital mode". This method could reach a spatial resolution of $\sim 1 \mathrm{~cm}$ which implies a maximum measurable energy of $10 \mathrm{GeV}$ [10]. As the interesting energy region for atmospheric neutrino oscillations spans from $\sim 1 \mathrm{GeV}$ to tens of $\mathrm{GeV}$, it is important to improve the spatial resolution of the detector to push the saturation point as high as possible. For this purpose, a second analysis was performed with the streamer tubes used in "drift mode". To check the electronics and the feasibility of the analysis, two tests were performed at the CERN PS-T9 and SPS-X7 beams. The space resolution achieved is $\simeq 3 \mathrm{~mm}$, a factor 3.5 better than in the first analysis. For each muon, seven MCS sensitive variables were given in input to a Neural Network (NN) previously trained to estimate the muon energy with MC events of known energy crossing the detector at different zenith angles. The method allowed to separate the upthroughgoing muons in 4 subsamples corresponding to average neutrino energies of 12, 20, 50 and $102 \mathrm{GeV}$, respectively. The comparison of the 4 zenith angle distributions with the predictions of the no oscillations MC shows a disagreement at low energies (where there is a deficit of vertical events), while the agreement is restored at the higher neutrino energies. The distribution of the ratios $R=\left(\right.$ Data $\left./ M C_{n o o s c}\right)$ obtained by this analysis is plotted in Fig. 5 as a function of $\log _{10}\left(L / E_{\nu}\right)$ [3,11]. The black points with error bars are the data; the vertical extent of the 


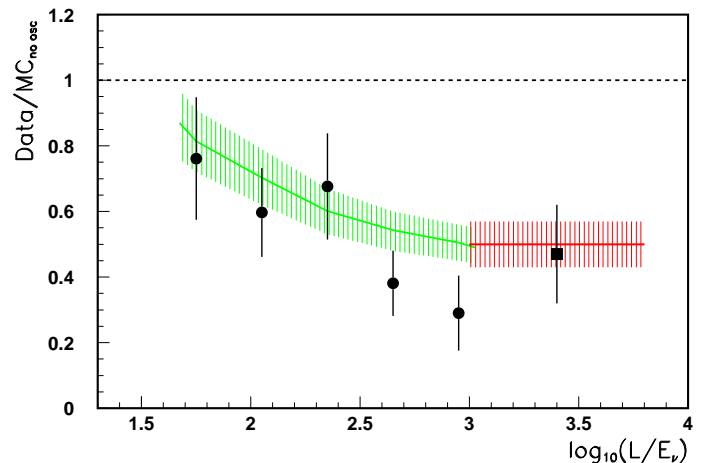

Figure 5. Data/MC vs $L / E_{\nu}$ for upthrougoing muons (black circles) and for semicontained up- $\mu$ (black square). The muon energy was estimated by MCS and $E_{\nu}$ by MC methods. The shaded region represents the uncertainty in the MC prediction assuming $\sin ^{2} 2 \theta=1$ and $\Delta m^{2}=0.0025 \mathrm{eV}^{2}$. The horizontal dashed line at Data/MC $=1$ is the expectation for no oscillations.

shaded areas represents the uncertainties on the MC predictions for $\nu_{\mu} \rightarrow \nu_{\tau}$ oscillations with maximal mixing and $\Delta m^{2}=2.5 \cdot 10^{-3} \mathrm{eV}^{2}$. The horizontal dashed line is the expectation without oscillations. The black square point was obtained from the low energy IU sample.

\section{II.4 Low energy data}

The Internal Upgoing (IU) muons come from $\nu_{\mu}$ interactions in the lower apparatus; for these events two scintilla-

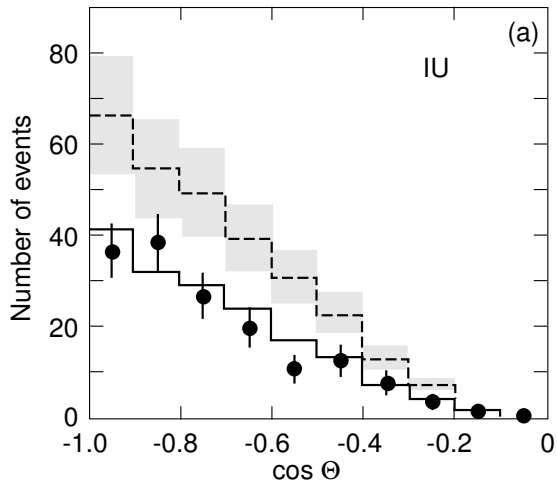

tion counters are intercepted; the T.o.F. is applied to identify upward going muons. The average parent neutrino energy for these events is $4.2 \mathrm{GeV}$. For neutrino oscillations one expects a reduction by about a factor of two in the flux, without any distortion in the shape of the angular distribution. This is what is observed in Fig. 6a, [12].

The upstopping muons (UGS) are due to external $\nu_{\mu}$ interactions yielding upgoing muons stopping in the detector. The semicontained downgoing muons (ID) are due to $\nu_{\mu}$ induced downgoing muon tracks with vertex in the lower MACRO (Fig. 1). The two types of events are identified by topological criteria; the lack of time information prevents to distinguish the two sub-samples. An almost equal number of UGS and ID events is expected. In case of oscillations, the flux of the UGS muons should be reduced by $50 \%$; no reduction is instead expected for the semicontained downgoing events (coming from neutrinos with path lengths of $\sim 20 \mathrm{~km}$ ); therefore one expects a global reduction of $25 \%$.

The number of events and the angular distributions are compared with MC predictions in Table 1 and Figs. 6a,b. The data show a uniform deficit for the whole angular distribution with respect to predictions, $\sim 50 \%$ for IU, $75 \%$ for ID + UGS; there is good agreement with the predictions based on neutrino oscillations with the parameters obtained from the upthroughgoing muons. The average value of the double ratio $R=(\text { Data } / M C)_{I U} /(\text { Data } / M C)_{I D+U G S}$ over the measured zenith angle range is $R \simeq 0.77 \pm 0.07$; the error includes statistical and theoretical uncertainties; $R=1$ is expected in case of no oscillations.

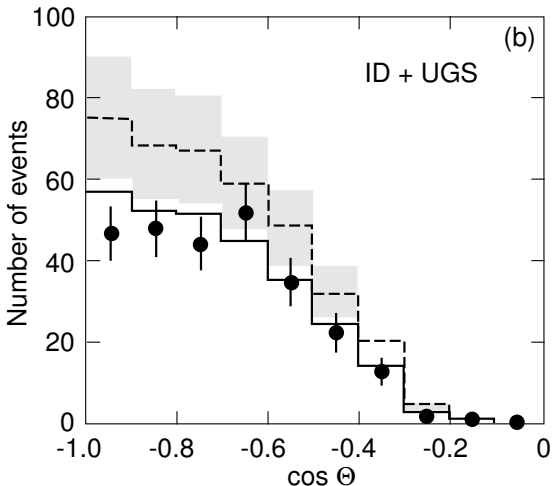

Figure 6. Zenith distributions for (a) the upsemicontained (IU) and (b) the upstopping plus the downsemicontained (UGD+ID) events. The black points are the data, the dashed lines at the center of the shaded regions correspond to MC predictions assuming no oscillations. The full line is the expectation for $\nu_{\mu} \rightarrow \nu_{\tau}$ oscillations.

\begin{tabular}{cccc}
\hline & Events & MC-No oscillations & $R=\left(\right.$ Data $\left./ M C_{\text {noosc }}\right)$ \\
\hline Up throughgoing & 809 & $1122 \pm 191$ & $\left(0.721 \pm 0.026_{\text {stat }} \pm 0.043_{\text {sys }} \pm 0.123_{\text {th }}\right)$ \\
\hline Internal Up & 154 & $285 \pm 28_{\text {sys }} \pm 71_{\text {th }}$ & $\left(0.54 \pm 0.04_{\text {stat }} \pm 0.05_{\text {sys }} \pm 0.13_{\text {th }}\right)$ \\
\hline Up Stop + In Down & 262 & $375 \pm 37_{\text {sys }} \pm 94_{\text {th }}$ & $\left(0.70 \pm 0.04_{\text {stat }} \pm 0.07_{\text {sys }} \pm 0.17_{\text {th }}\right)$ \\
\hline
\end{tabular}

Table 1. Summary of MACRO $\nu_{\mu} \rightarrow \mu$ events in $-1<\cos \theta<0$ (after background subtraction) for oscillation studies. For each topology (see Fig. 1) the number of measured events, the MC prediction for no-oscillations and the ratio (Data/MC-no osc) are given [2, 3, 12]. 


\section{Search for Astrophysical Sources of High Energy Muon Neutrinos}

High energy $\nu_{\mu}$ are expected to come from several galactic and extragalactic sources. Neutrino production requires astrophysical accelerators of charged particles and some kind of astrophysical beam dumps.

The excellent angular resolution of our detector allowed a sensitive search for upgoing muons produced by neutrinos coming from celestial point sources, with a negligible atmospheric neutrino background. An excess of events was searched for around the positions of known sources in $3^{\circ}$ (half width) angular bins. This value was chosen so as to take into account the angular smearing due to multiple muon scattering in the rock below the detector and by the energyintegrated angular distribution of the scattered muon, with respect to the neutrino direction.

The pointing accuracy of the detector was checked with the observation of the shadow of primary cosmic rays (and thus on downgoing muons) by the Moon and the Sun [13]. It was also checked with the study of the correlations between muons detected by MACRO and extensive air showers detected by the EASTOP experiment located above the Gran Sasso massif [14].

In a total lifetime of 6.16 y we obtained a total of 1356 events, see Fig. 7a [3, 13]. The $90 \%$ c.l. upper limits on the muon fluxes from specific celestial sources lay in the range $10^{-15}-10^{-14} \mathrm{~cm}^{-2} \mathrm{~s}^{-1}$, see Fig. $7 \mathrm{~b}$. The solid line is our sensitivity vs declination. Notice that we have two cases, GX339-4 $\left(\alpha=255.71^{\circ}, \delta=-48.79^{\circ}\right)$ and Cir X-1 $\left(\alpha=230.17^{\circ}, \delta=-57.17^{\circ}\right.$ ), with 7 events: in Fig. $7 \mathrm{~b}$ they are considered as background, therefore the upper flux limits are higher; but they could also be indications of signals $[3,15]$. We searched for time coincidences of our upgoi muons with sources of $\gamma$-ray bursts as given in the BATSE 3B and 4B catalogues, for the period from April 91 to December 2000 [15]. No statistically significant time correlation was found.

We have also searched for a diffuse astrophysical neutrino flux, for which we establish a flux upper limit at the level of $1.5 \cdot 10^{-14} \mathrm{~cm}^{-2} \mathrm{~s}^{-1}[16]$.

\section{Indirect Searches for WIMPs}

Weakly Interacting Massive Particles (WIMPs) could be part of the galactic dark matter; they could be intercepted by celestial bodies, slowed down and trapped in their centers, where WIMPs and anti-WIMPs could annihilate and yield $\nu_{\mu} \rightarrow$ upthroughgoing muons. The annihilations in these celestial bodies would yield neutrinos of $\mathrm{GeV}-\mathrm{TeV}$ energy, in small angular windows from their centers. For the Earth we have chosen a $15^{\circ}$ cone around the vertical: we find 863 events. The MC expectation for atmospheric $\nu_{\mu}$ without oscillations gives a larger number of events. We set a conservative flux upper limit assuming that the measured number of events equals the expected ones. We obtain the $90 \%$ c.l. limits for the upgoing muon flux shown in Fig. 8a (it varies from 0.8 to $0.510^{-14} \mathrm{~cm}^{-2} \mathrm{~s}^{-1}$ ) [17, 3]. If the WIMPs are identified with the smallest neutralino mass, our limits may be used to constrain the neutralino mass, following the model of ref. [18], Fig. 8a.

A similar procedure was used to search for muon neutrinos from the Sun, using 10 search cones from $3^{\circ}$ to $30^{\circ}$. In the absence of statistically significant excesses the muon flux upper limits are at the level of $1.5-2 \cdot 10^{-14} \mathrm{~cm}^{-2} \mathrm{~s}^{-1}$. The limits are shown in Fio $8 \mathrm{~h}$ as a function of the WIMP

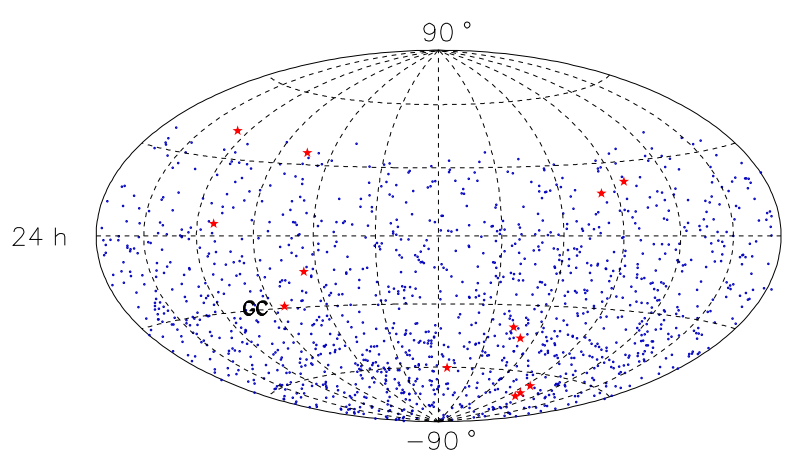

(a)

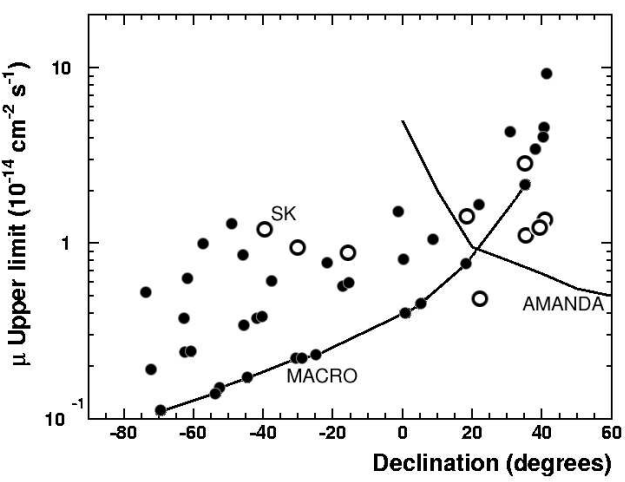

(b)

Figure 7. High energy $\nu_{\mu}$ astronomy. (a) Upgoing muon distribution in equatorial coordinates (1356 events). (b) The black points are the MACRO $90 \%$ c.l. upwardgoing muon flux limits as a function of the declination for 42 selected sources. The solid line refers to the limits obtained for those cases for which the atmospheric neutrino background was zero. The limits obtained by the SK (open circles) and AMANDA (thin line) experiments are quoted. 


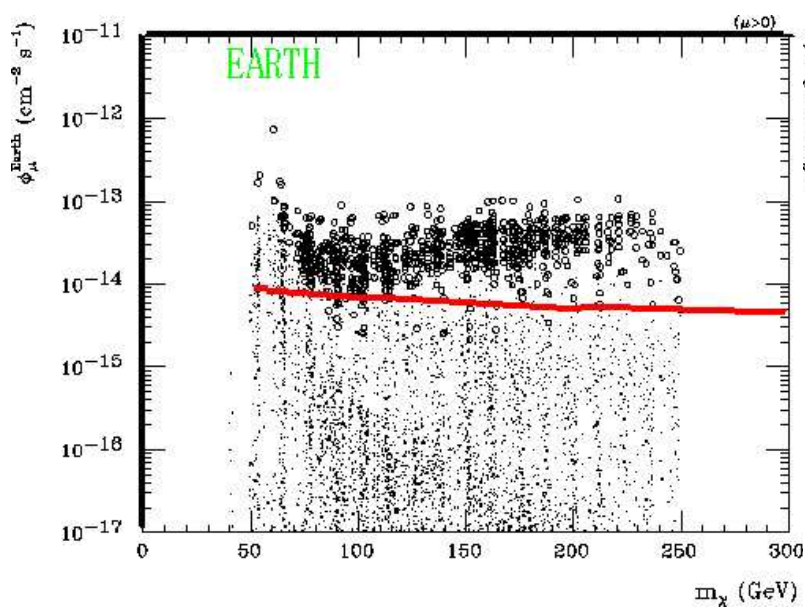

(a)

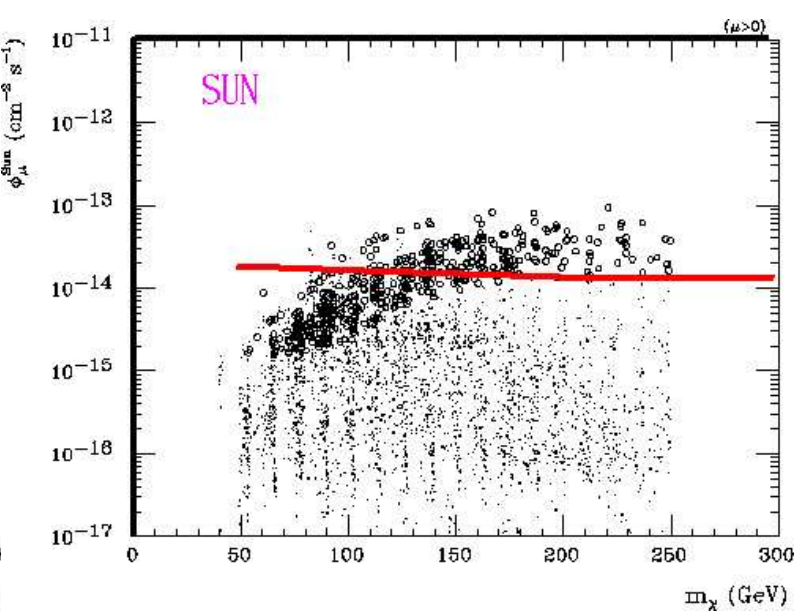

(b)

Figure 8. (a) The solid line is the MACRO upwardgoing muon flux upper limit (90\% c.l.) from the Earth plotted vs neutralino mass $m_{\chi}$. (b) The same as in (a) but for upwardgoing muons from the Sun. Each dot is obtained varying model parameters. The open circles indicate models excluded by direct measurements assuming a local dark matter density of $0.5 \mathrm{GeVcm}^{-3}$.

\section{Neutrinos from Stellar Gravita- tional Collapses}

A stellar gravitational collapse (GC) of the core of a massive star is expected to produce a large burst of all types of neutrinos and antineutrinos with energies of $7-30 \mathrm{MeV}$ and with a duration of $<10 \mathrm{~s}$. The $\bar{\nu}_{e}$ 's can be detected via the process $\bar{\nu}_{e}+p \rightarrow n+e^{+}$in the liquid scintillator. About $100 \div 150 \bar{\nu}_{e}$ events should be detected in our $580 \mathrm{t}$ scintillator for a stellar collapse at the center of our Galaxy.

We used two electronic systems to search for bursts of $\bar{\nu}_{e}$ 's from stellar gravitational collapses. The first system was based on the dedicated PHRASE trigger, the second one was based on the ERP general trigger. Both had energy thresholds of $\sim 7 \mathrm{MeV}$ and recorded pulse shape, charge and timing informations. Immediately after a trigger, the PHRASE system lowered its threshold to $1 \mathrm{MeV}$, for a duration of $800 \mu \mathrm{s}$, in order to detect (with $\mathrm{a} \simeq 25 \%$ efficiency) the $2.2 \mathrm{MeV} \gamma$ released in the reaction $n+p \rightarrow$ $d+\gamma_{2.2 \mathrm{MeV}}$ induced by the neutron produced in the primary process.

A redundant supernovae alarm system was in operation, alerting immediately the physicists on shift. We defined a general procedure to alert the physics and astrophysics communities in case of an interesting alarm. Finally, a procedure to link the various supernova observatories around the world was set up [3, 19].

The effective MACRO active mass was $\sim 580 \mathrm{t}$; the lifetime fraction was $\simeq 97.5 \%$; we kept always ruming at least 1-2 supermodules. No stellar gravitational collapses were observed in our Galaxy from the beginning of 1989 to the end of 2000 [3][19].

\section{Conclusions}

The MACRO detector took data from 1989 to the end of the year 2000 and it obtained important results in all the items listed in the proposal, in particular:

- Atmospheric neutrino oscillations. Analyses of different event topologies at different energies, the exploitation of the muon Coulomb multiple scattering in the detector give strong support to the hypothesis of $\nu_{\mu} \rightarrow \nu_{\tau}$ oscillations with $\Delta m^{2}=0.0025 \mathrm{eV}^{2}$ and maximal mixing. We are presently making the final global analysis of all our data.

- High energy muon neutrino astronomy. MACRO has been highly competitive with other underground experiments thanks to its large acceptance and good angular accuracy. It has been limited by its lifetime and the size of the detector.

- Search for bursts of $\bar{\nu}_{e}$ from stellar gravitational collapses. MACRO was sensitive to supernovae events in our Galaxy, it started the SN WATCH system, and for a certain time it was the only detector in operation.

- Sensitive indirect searches have been carried out for possible Dark Matter candidates like the neutralinos, looking for upgoing muons from the center of the Earth and from the Sun.

It may be pointed out that MACRO also obtained the best flux upper limits for GUT Magnetic Monopoles over the widest $\beta$ range [20].

\section{Acknowledgments}

I thank the members of the MACRO Collaboration for their cooperation; in particular I acknowledge the discussions and the help from the members of the Bologna group, 
in particular Drs. Y. Becherini, M. Giorgini and M. Spurio. I thank Ms Anastasia Casoni for typing and correcting the manuscript.

\section{References}

[1] S. Ahlen et al., Nucl. Instr. Meth. Phys. Res. A 324, 337 (1993); M. Ambrosio et al, Nucl. Instr. Meth. Phys. Res. A 486, 663 (2002); hep-ex/0207043.

[2] S. Ahlen et al., Phys. Lett. B 357, 481 (1995); Phys. Lett. B 434, 451 (1998).

[3] M. Ambrosio et al., hep-ex/0206027.

[4] V. Agrawal et al., Phys. Rev. D 53, 1314 (1996).

[5] M. Gluck et al., Z. Phys. C 67, 433 (1995).

[6] G. Feldman and R. Cousins, Phys. Rev. D 57, 3873 (1998).

[7] SK Coll., Y. Fukuda et al., Phys. Rev. Lett. 81, 1562 (1998); Phys. Rev. Lett. 85, 3999 (2000); hep-ex/0105023.

[8] Soudan 2 Coll., W.W.M. Allison et al., Phys. Lett. B 449, 137 (1999).
[9] M. Ambrosio et al., Phys. Lett. B 517, 59 (2001).

[10] D. Bakari et al., hep-ex/01050871.

[11] M. Ambrosio et al., Nucl. Instr. Meth. Phys. Res. A ??????, hep-phys/0203018.

[12] M. Ambrosio et al., Phys. Lett. B 478, 5 (2000); hepex/01120021.

[13] M. Ambrosio et al., Phys. Rev. D 59, 012003 (1999).

[14] EASTOP-MACRO Coll., M. Aglietta et al., Phys. Lett. B 337, 376 (1994).

[15] M. Ambrosio et al., Astrophys. J. 546, 1038 (2001).

[16] M. Ambrosio et al., astro-phys/0203181.

[17] M. Ambrosio et al., Phys. Rev. D 60, 082002 (1999).

[18] A. Bottino et al., Proceedings of the Ringberg Euroconf. New Trends in Neutrino Physics, Ringberg Castle, Germany, 1998, edited by B. Kniel, World Scientific, Singapore.

[19] M. Ambrosio et al., Astroparticle Phys. 8, 123 (1998).

[20] M. Ambrosio et al., Eur. Phys. J. C 25, 511 (2002) hepex/0207020; Eur. Phys. J. C 26, 163 (2002), hep-ex/0207024. 